\title{
¿Cómo quedamos los pacientes frente a la nueva reforma del sistema de salud?
}

Where are patients in front of the new health reform?

- Laura Ramírez', Adriana María Garzón

'Fundación Simmon (Bogotá, Colombia).

A diario, los colombianos tenemos que enfrentar la crisis de un sistema de salud en donde quienes somos pacientes cada vez nos encontramos con más y más problemas. Las soluciones ni se vislumbran $y_{\text {, }}$ mientras tanto, son nuestras vidas las que se ponen en juego. Ni qué decir de las barreras de acceso que existen y que son el día a día de quienes necesitamos de un servicio de salud digno. La problemática es clara: inoportunidad en la atención, demora en autorizaciones, exceso de trámites administrativos, falta de acceso a los especialistas y desabastecimiento de medicamentos, para citar algunas de las muchas complicaciones que aquejan a nuestra población. Sin embargo, las autoridades en salud parecen no hallar correctivos acertados, rápidos ni efectivos que mejoren la atención en salud de los miles de colombianos que hoy son pacientes. Y mucho menos, se está pensando en tomar medidas que se traduzcan en beneficio para nosotros.

Así lo demuestra la intención del Ministerio de Salud y Protección Social, que consideró que la alternativa legislativa era la mejor, por eso, a finales del año 2012, presentó ante el Congreso de la República un proyecto de ley estatutaria, donde se pretendía definir el alcance del derecho a la salud. Pero en este proyecto no se contenía ningún cambio estructural del sistema que nos diera luces para pensar que la situación realmente cambiaría.

Ya a inicios del 2013, el Ministerio de Salud anunció que, ante la grave y evidente crisis por la que atraviesa el sistema de salud, lo que se requería era una reforma, vía ley ordinaria. Por ello se empezó a notificar los cambios en los que se estaba trabajando y que se consideraron la mejor opción. Frente a los anuncios públicos, los diferentes actores que conforman el sistema de salud comenzaron a reaccionar y a cuestionar si de lo que estaba hablando el Ministerio realmente era lo que necesitaba el país. ¿Darían respuesta a las peticiones que en los últimos años todos los colombianos hemos venido exigiendo, para que exista un gran cambio y la salud sea efectivamente un derecho?

Finalmente, el 19 de marzo, fueron expuestos los proyectos de ley a los que el gobierno le apuesta para mejorar la salud de los colombianos. Uno de ellos es una nueva ley estatutaria, conformada por 14 artículos trabajados por la Gran Junta Médica Colombiana, que busca garantizar la salud como un derecho humano constitucional y fundamental, logrando que el Estado sea el responsable de garantizar, propiciar, facilitar y tutelar el derecho a la salud para todos los colombianos desde la procreación hasta la muerte. Este proyecto reemplaza al que el Ministerio de Salud había presentado en diciembre del año pasado y debe hacer su trámite en la comisión primera.

El otro proyecto presentado y radicado en el Senado de la República fue un proyecto de ley ordinaria trabajado por el Ministro de Salud. Este es el Proyecto de Ley 210 de 2013, "por medio del cual se redefine el sistema general de seguridad social en salud y se dictan otras disposiciones". Dicho proyecto, que consta de 9 capítulos y 67 artículos, y que hará su trámite legislativo por las comisiones séptimas de Senado y Cámara, pretende mostrarnos cómo funcionará nuestro nuevo sistema de salud. A continuación explicaremos algunos de los temas que nos inquietan:

- El proyecto de ley no presenta una reforma estructural al actual sistema de salud, y no es un cambio de fondo a la Ley 100 de 1993. Vemos que se mantiene el modelo de aseguramiento, tratando a la salud como un 'servicio público', mas no como un derecho fundamental; se mantienen los dos regímenes: contributivo y subsidiado; sigue la fragmentación de los servicios y, sobre atención primaria en salud, se 
han quedado cortos. Es por eso por lo que, desde el inicio, el proyecto plantea que solo se redefinirá el sistema que ahora conocemos.

- Este proyecto de ley, aunque expone alrededor de 20 principios, sobre los cuales se enmarca, nos hace pensar que se trata de un proyecto garantista que incluye moedelos con los que todos nos identificamos, como solidaridad, equidad, calidad e integralidad. Pero hay que ser cuidadoso cuando se enmarcan otros principios, como la universalidad o corresponsabilidad, en donde se retoma el autocuidado. O uno de los que más polémica ha generado el principio de inembargabilidad de los recursos en salud, que nos dice que "los recursos del sistema destinados a las prestaciones individuales son públicos hasta que se transfieren desde Salud Mía a los agentes del sistema", lo que significa que, una vez el fondo único que se crea gire los recursos de salud a los diferentes agentes del sistema, ya no se les podrá hacer ningún seguimiento, ni se podrá vigilar ni controlar el manejo de los recursos públicos que son destinados únicamente para atender los servicios de salud, porque pasarán a ser privados y, de esta manera, se deja abierta la puerta para que los distintos actores inviertan sin responsabilidad. Como pacientes nos preocupa la portabilidad nacional y, sobre todo, el que sigan anteponiendo el criterio de sostenibilidad fiscal al de la garantía del derecho a la salud.

- Lo más novedoso y quizá de beneficio para nosotros se da en la creación de un único fondo, una unidad de gestión, llamada Salud Mía (artículo 5º), que se encargará de afiliar a la población, recaudar las cotizaciones, administrar los recursos a su cargo y realizar los pagos, giros o transferencias. Con este cambio, se liquidará el Fondo de Solidaridad y Garantía (Fosyga), eliminando así el manejo de los dineros de la salud por parte de los intermediarios o, mejor, de las EPS, puesto que los pagos se harán directamente a los prestadores (artículo 27).

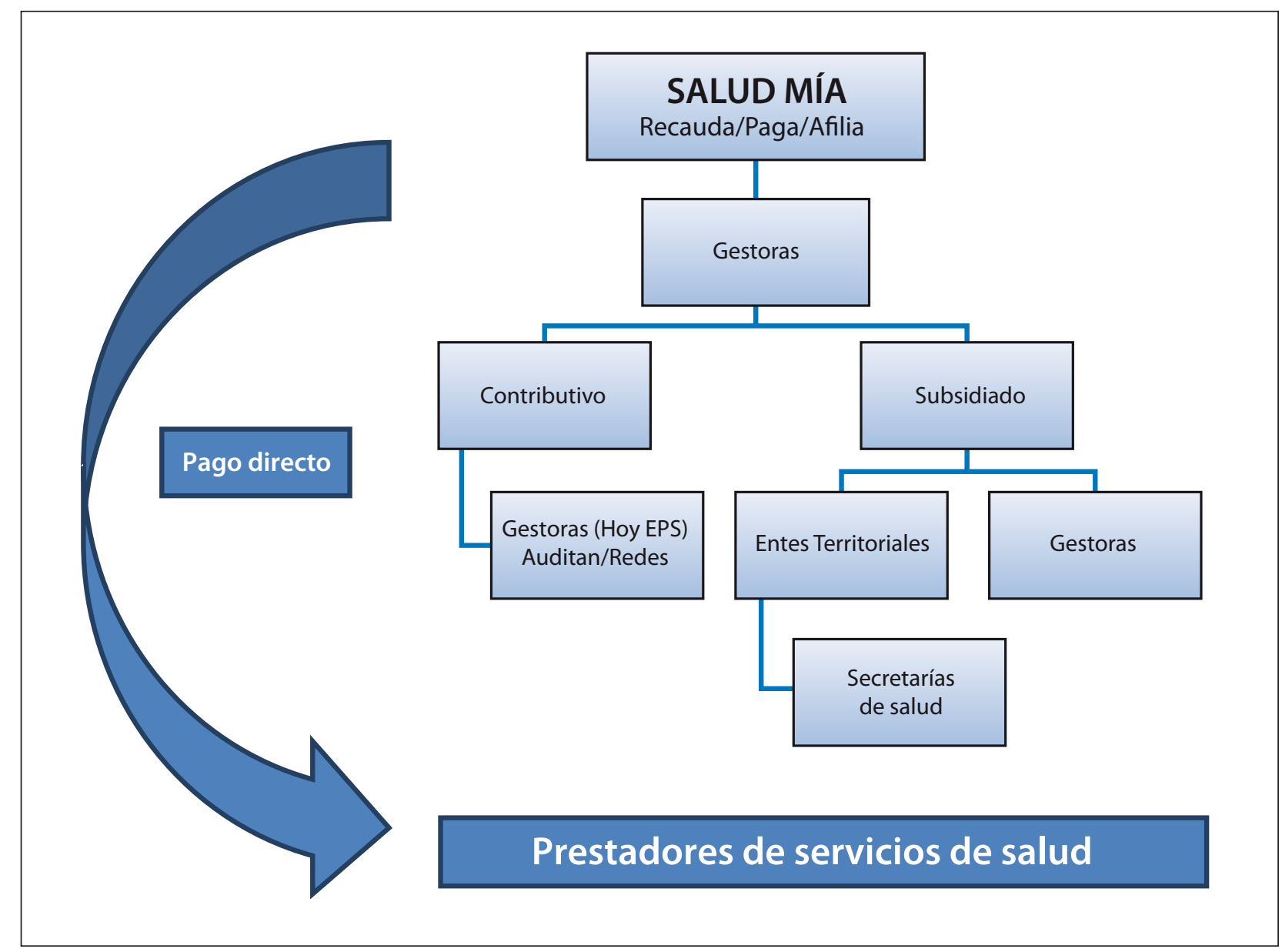

Figura 1. Conformación del sistema de salud (Colombia 2013). 
Este fondo público estaría adscrito al Ministerio de Salud y sería vigilado por la Superintendencia Financiera. Desafortunadamente, en el órgano de dirección de esta unidad de gestión, es decir, en su junta directiva, no habrá participación más que de representantes del gobierno (preside el Ministro de Salud y Protección Social, y lo acompañan el Ministro de Hacienda y Crédito Público, y un designado del Presidente de la República). La figura 1 resume la estructura del nuevo modelo para el sistema de salud (Colombia 2013).

- Respecto al plan de beneficios de salud, antes llamado POS, ahora se nombrará como Mi Plan, en teoría será más integral; así reza el artículo 14: "Corresponde a los servicios y tecnologías aprobadas para su uso en el país y requeridas para atención en salud. Mi Plan garantizará la cobertura de los servicios y tecnologías para todas las patologías. Se establecerá un listado de servicios y tecnologías excluidos de Mi Plan, de acuerdo con los criterios definidos en el capítulo III". Para nosotros, no es claro cómo se conformarán y cómo se actualizarán estos planes: el que incluye todo lo que está en Mi Plan y en el de las exclusiones.

A pesar de que el artículo 15 habla de garantizar la participación social en el proceso de priorización y posterior definición de las exclusiones, nos inquieta que queden fuera aquellos tratamientos que no curan pero que sí mantienen a nuestros pacientes crónicos con calidad de vida por años. A estas tecnologías o tratamientos el sistema los consideraría no razonables, por lo que tendrían que ser cubiertos por los pacientes.

- En cuanto a la prestación del servicio, se establece sin mucha claridad cómo será la coordinación para esas acciones de salud pública entre los gestores, responsables de la organización y gestión de las prestaciones individuales y las entidades territoriales (artículo 20). La conformación y organización de redes prestadoras de servicios de salud, igualmente, estará a cargo de los gestores. Nos inquieta la división entre prestadores de servicios de salud primarios y prestadores de servicios de salud complementarios, así como la creación de las nuevas áreas de gestión sanitaria, que el Ministerio de Salud y Protección Social integraría con redes de prestadores de servicios de salud gestionadas por la administradora. El funcionar solo en una región en particular podría constituirse en una nueva barrera para los pacientes que salgan del área de cobertura.

- El anuncio que hizo el ministerio acerca de que las EPS iban a acabarse nos tomó por sorpresa. Pero descubrimos que no desapareceran, sino que podrán transformarse en estas nuevas y llamativas organizaciones denominadas gestores de servicios de salud, los cuales son "personas jurídicas de carácter público, privado o mixto, vigilados por la Superintendencia Nacional de Salud, responsables de operar una red de prestadores de servicios de salud dentro de un área de gestión sanitaria para garantizar las prestaciones individuales de Mi Plan". "Los gestores de servicios de salud privados, incluidos los programas de salud de las cajas de compensación, deben constituirse como sociedades anónimas" (artículo 26). Tales gestores tendrán un órgano colegiado de dirección, pero, sin lugar a dudas, son ellos los reales protagonistas del nuevo sistema. Dentro de sus múltiples funciones, se destaca todo lo relacionado con las redes prestadoras de servicios: la conformación, su organización, gestión y operación. También, la suscripción de contratos, la coordinación con las entidades territoriales de las acciones de salud pública, los cobros de copagos y cuotas moderadoras a los pacientes, y el auditar los pagos para autorizar los giros directos que desde Salud Mía se harían a los prestadores. Pensaríamos que los gestores pueden llegar a adueñarse de las IPS.

Nos asalta la duda frente a la tan cuestionada integración vertical. Esperemos que no supere el luchado 30\% establecido en la actualidad (artículo 28), y, para nuestra decepción, vemos con tristeza los artículos 32 y 33. En el artículo 32 se menciona que "Está prohibida la integración vertical de gestores de servicios de salud y prestadores de servicios de salud salvo en la prestación primaria de servicios. Los pacientes se quedarán entonces en la red básica de atención, porque de ninguna manera les convendrá remitirlos al especialista, ya que no recibirían ningún pago". Y en el artículo 33 los estimula diciendo: "Cada gestor de servicios de salud en una determinada área de gestión sanitaria tendrá derecho a reconocimientos económicos y pagos por parte de Salud Mía: a) una suma fija anual para financiar gastos de administración, definida por el Ministerio de Salud y Protección Social con base en 
el número de usuarios, la cual será girada en cuotas mensuales; b) Un valor per cápita ajustado por riesgo. Este se dividirá en: (i) una fracción para cubrir parcialmente el costo de las prestaciones individuales de $\mathrm{Mi}$ Plan, y (ii) la fracción restante condicionada al logro de acciones o resultados en salud". Para nosotros, es clara la posición dominante de estos nuevos actores, que llegan con los mejores beneficios.

Parece ser que nuestros legisladores han olvidado las irregularidades cometidas en años anteriores por las empresas promotoras de salud, quienes pasarán a ser los nuevos gestores. Acaso no es diciente el estudio La tutela y el derecho a la salud del año 2011, preparado por la Defensoría del Pueblo, en donde tenemos datos recientes y relevantes que nos muestran que el $72,5 \%$ de las tutelas en salud (interpuestas por tratamientos, medicamentos, cirugías y citas médicas especializadas) se dirigieron contra las EPS que administran los regímenes contributivo y subsidiado; que las cuotas moderadoras y copagos siguen siendo una gran barrera de acceso a los servicios de salud; y que aun la unificación del POS de los regímenes contributivo y subsidiado no es garantía de la disminución de las tutelas por servicios POS. Y este insólito dato que nos llama con gran poder la atención: el $67,81 \%$ de las solicitudes en las tutelas en salud se encuentra incluido dentro del POS y es el régimen subsidiado el que presenta el mayor número de ellas. Nos preguntamos, por qué la han ignorado, por qué no se han acatado recomendaciones que se vienen haciendo desde el año 2010, algunas de ellas incluso exhortando al cumplimiento de sentencias emitidas por la Corte Constitucional, como la T-760 del año 2008.

Entonces no era necesario fortalecer a la Superintendencia de Salud para que ejerza sus funciones de inspección, vigilancia y control, adoptando medidas ejemplarizantes sobre aquellas entidades que de manera reiterada han vulnerado el derecho a la salud. ¿Por qué no pensar en una Superintendencia Nacional de Salud más descentralizada, con mayores recursos, que sea independiente y autónoma, y que esté separada del Ministerio de Salud y Protección Social?

- Con relación a la cobertura complementaria en salud (artículo 37), lo primero que pensamos es que esta deberá ser pagada por los pacientes $y$, en detrimento de su bolsillo, serán las empresas de medicina prepagada, las compañías aseguradoras $y$, de nuevo, los gestores de servicios de salud los aventajados.

- Aunque esperábamos mucho más en este capítulo que es de vital importancia para nosotros como pacientes, al menos nos alegra saber que por fin están pensando en que la Superintendencia Nacional de Salud tenga un poco más de agilidad en sus procesos sancionatorios. Para terminar, no quisiéramos desconocer las buenas intenciones que tiene el gobierno respecto a los plazos para que todo lo anteriormente expuesto se haga operativo. En un período de máximo dos años, parece imposible la implementación de este amplio y complejo articulado.

- Tampoco quisiéramos finalizar sin exponer nuestra preocupación y la de muchos colombianos que en años anteriores, impotentes, han visto desaparecer millonarias sumas de dinero en manos de algunos. ¿Podrá Colombia superar este gran reto? Pensemos en la salud con la mano en el corazón y actuemos aquí y ahora, porque el tiempo es corto y los intereses son muchos. Infortunadamente es la política que destrona a quien debería ser el centro de este sistema: el colombiano, un ser humano que en cualquier momento pasa de estar sano a ser un paciente con cáncer. 